\title{
A Rotating Stellar Collapse Model for Supernova 1987a
}

TAKashi NaKamura

\author{
Department of Physics, Kyoto University, Kyoto 606, Japan \\ Masataka Fukugita
}

Research Institute for Fundamental Physics

Kyoto University, Kyoto 606, Japan

It is shown that the bunch structure of the Kamiokande neutrino events associated with SN1987a can be naturally understood, if one assumes that the core of the progenitor star was rotating moderately with $q\left(\equiv J c / G M^{2}\right) \approx 3$ with $\mathbf{J}$ the total angular momentum and $M$ the gravitational mass of the core.

We assume that the presence of the observed gap, at least that between the second and the third bunch, is real and consider its implications in the dynamics of the core collapse. Let us define a nondimensional angular momentum $q\left(\equiv J c / G M^{2}\right)$ with $\mathrm{J}$ the total angular momentum and $\mathrm{M}$ the gravitational mass of the core. We assume that the value of $\mathrm{q}$ for the core of SN1987a was about 3. Then we expect that the effect of the angular momentum plays an important role when the size of the core $r$ shrinks to $<10^{7} \mathrm{~cm}$. In the spherically symmetric collapse model, the size of the unshocked homologous core is of the order of $10^{7} \mathrm{~cm}$. Hence we expect that the core at the bounce is essentially governed by the spherically symmetric dynamics. To verify this point we may refer to the simulation by Symbalisty. ${ }^{1}$ Our model almost corresponds to Model ROT2 in which one obtains a neutrinosphere with a roughly spherical radius of approximately $50 \mathrm{~km}$. We then expect that the infalling nonhomologous matter onto the core will liberate a gravitational energy of the order of $10^{52}$ ergs.

In the spherically symmetric model, the core enters the Kelvin-Helmholtz contraction phase , and its radius is expected to be $10^{6} \mathrm{~cm}$ within $1 \mathrm{sec}$ or so after the bounce ${ }^{2}$ The evolution of a rotating star is different. The contraction takes place mainly along the rotational axis. This is clearly seen in the case with Model ROT2. In this Kelvin phase the released gravitational energy is at most $\frac{(\pi / 2-1) \cdot 3 \cdot G M^{2}}{5 r} \approx 2 \cdot 10^{52}$ ergs. Using the cooling rate calculated by Burrows and Lattimer $^{2}$, one finds that the core becomes a disk with thickness $\mathrm{d} \approx 2 \cdot 10^{6} \mathrm{~cm}$ within $0.5 \mathrm{sec}$. We then have a rotating disk-like proto neutron star with an aspect ratio $r / d \approx 5$. Such a thin disk is known to be gravitationally unstable irrespective of the equation of state. It fragments into $(r / d)^{2} / \pi^{2}$ pieces in a free fall time scale $\approx 0.01 \mathrm{sec}$, because the most unstable mode has a wave length $2 \pi d$. Three dimensional numerical simulations of collapse of rotating isothermal clouds also support this result." In our case we will have two or three fragments. Since the specific spin angular momentum is reduced by a factor of 3 to 5 , the effect of angular momentum is not important for the Kelvin contraction of each fragment. Then the binding energy of neutron 
stars $\approx 10^{53}$ ergs will be liberated after all of the order of one second according to the cooling rate in Ref.2. We consider that the second neutrino bunch corresponds to this phase.

Let us assume for simplicity that a binary-like system with masses $m_{1}$ and $m_{2}$ with a separation distance $\mathbf{r}$ is formed as a result of the fragmentation. Then the distance $r$ decreases due to the emission of gravitational radiation at the rate of $\dot{r}=-7.7 \cdot 10^{5} \mathrm{~cm} / \mathrm{sec}$. $\frac{f \cdot\left(m_{1} / 0.7 M_{\odot}\right) \cdot\left(m_{2} / 0.7 M_{\odot}\right) \cdot\left(\left(m_{1}+m_{3}\right) / 1.4 M_{\odot}\right)}{\left(r / 10^{7} \mathrm{~cm}\right)^{3}}$, with $f$ the efficiency factor. Equation $(2)$ for $f=1$ is derived by assuming that two bodies are point particles. As a value of $f$ we adopt $f=0.1 \approx 0.5$ to take into account non-point like effects because an extended object is a weaker emitter of the gravitational radiation due to phase cancellation effects ${ }^{5,6}$ Now the characteristic time of the decrease of the separation $\mathbf{r}$ due to the emission of the gravitational radiation is given by $t=9 s e c \cdot(f / 0.3)^{-1} \cdot \frac{\left(r / 10^{7} c m\right)^{4}}{\left(m_{1} / 0.7 M_{\odot}\right) \cdot\left(m_{2} / 0.7 M_{\odot}\right) \cdot\left(\left(m_{1}+m_{3}\right) / 1.4 M_{\odot}\right)}$.

If the two fragments have almost equal mass $0.7 M_{\odot}$, then two proto neutron stars will collide after $9 \mathrm{sec}$ with a subsonic speed $2.2 \times 10^{8} \mathrm{~cm} / \mathrm{sec}$. Eventually a rapidly rotating neutron star of mass $1.4 M_{\odot}$ will be formed and the difference of the binding energy up to $10^{53}$ ergs between proto neutron stars and a final single neutron star should be liberated as neutrinos, gravitational radiation and electromagnetic waves. Alternatively, if the two fragments have different masses, say, $0.9 M_{\odot}$ and $0.5 M_{\odot}$ then the binary system enters a stable mass stripping phase under the plausible parameters of mass loss and angular momentum loss . ' In a numerical calculation for the case of two neutron stars of mass $1.3 M_{\odot}$ and $0.8 M_{\odot}$ the integrated energy of the neutrino emission by the mass accretion is estimated to be $2 \cdot 10^{53}$ ergs with time duration about 2 sec. $^{7}$ We can estimate the average energy of neutrinos in the accretion phase to be $\approx 10 \mathrm{MeV}^{7}$. In any case we expect that a neutrino flux with total energy $\approx 10^{53} \mathrm{ergs}$ is liberated almost $10 \mathrm{sec}$ after from the first burst.

\section{REFERENCES}

1. E.D. Symbalisty, Asrtrophys. J. 285(1984),729.

2. A. Burrows and J.M. Lattimer, Asrtrophys. J. 307(1986) 178.

3. P. Goldreich and D. Lynden-Bell, Mon. Not. R. A. Soc. 130(1965) 97.

4. S.M. Miyama, S. Narita and C. Hayashi Asrtrophys. J. 279(1984) 621.

5. T. Nakamura and M. Sasaki, Phys. Letters 106b(1981) 69.

6. T. Nakamura and K. Oohara, Phys. Letters 98A(1983) 403.

7. J.P.A. Clark and D.M. Eardley, Astrophys. J. 215(1977) 311. 